\title{
Artificial Synapse and Neuron Combining the Ion-Sensitive Field-Effect Transistor and Memristor
}

\author{
Sungiu Choi ${ }^{1}$, Seohyun Kim ${ }^{1}$, Jungkyu Jang ${ }^{1}$, Gumho Ahn ${ }^{1}$, Jun Tae Jang ${ }^{1}$, Tae Jung Park ${ }^{2}$, Byung-Gook Park ${ }^{3}$, \\ Dong Myong Kim ${ }^{1}$, Sung-Jin Choi ${ }^{1}$, Seung Min Lee ${ }^{1}$, Hyun-Sun Mo ${ }^{1, * *}$, and Dae Hwan Kim ${ }^{1, *}$ \\ 1 School of Electrical Engineering, Kookmin University, Seoul 02707, Republic of Korea \\ 2 Department of Chemistry, Chung-Ang University, Seoul 06974, Republic of Korea \\ ${ }^{3}$ Department of Electrical and Computer Engineering, Seoul National University, Republic of Korea \\ "drlife@kookmin.ac.kr, ${ }^{* *} t y c h e @ k o o k m i n . a c . k r$
}

\begin{abstract}
:
Implementations of artificial synapse and neuron are presented by combining the silicon nanowire ionsensitive field-effect transistor (ISFET) and the indium-gallium-zinc-oxide (IGZO) memristor. Chemical and electrical operations of synapse are emulated by the $\mathrm{pH}$ sensor operation of ISFET and by the long-term potentiation of IGZO memristor, respectively. The concentration of hydrogen ions in electrolyte is successfully transformed via the voltage-controlled oscillator (VCO)-based neuron into the modulation of synaptic strength, i.e., the current of memristor. Proposed synapse and neuron show the feasibility of emulating the brain-inspired computing along with taking into accounts both chemical and electrical operation of synapse.
\end{abstract}

Key words: human-like sensory circuit, Silicon nano wire, $\mathrm{pH}$, memristor,

\section{Introduction}

Recently, great efforts have been made to overcome the limit of energy-efficiency of vonNeumann computing system by employing the human brain-inspired neuromorphic computing system. Here, the emulation of biological systems is an indispensable step toward the design and verification of large-scale integrated neuromorphic system and architecture. Noticeably, the chemical as well as the electrical function of synapse needs to be considered for systematic emulation. The latter means not only the long-term potentiation (LTP) and short-term depression (STD) but also the spike timing-dependent plasticity (STDP) in persepctive of spiking neural network [1] and while the former needs to detect the concentration of neurotransmitter agents, i.e., glutamate, $\gamma$-Aminobutyric Acid (GABA), and glycine, with the functionalization of their specific-binding receptors [2]. However, both the chemical and electrical synaptic behaviors have been seldom demonstrated in a single platform. In this work, artificial synapse and neuron are demonstrated by combining the silicon nanowire (SiNW) ion-sensitive fieldeffect transistor (ISFET) and the amorphous indium-gallium-zinc-oxide (IGZO) memristor.

\section{Experiment}

The biological system consisting of synapses and neurons is illustrated in Fig. 1(a) and correspondingly, artificial synapse and neuron are implemented in Fig. 1(b). The action potential at the presynaptic neuron is transformed into an ejected neurotransmitter. Then, this neurotransmitter diffuses and binds at the receptor in the postsynaptic neuron and converts to action potentials again. These chemical synapse is emulated by SiNW ISFET. Its fabrication details can be found in [3]. In addition, the electrical synapse is emulated by the two-terminal IGZO memristor. The neuron is implemented with VCO and amplifiers.
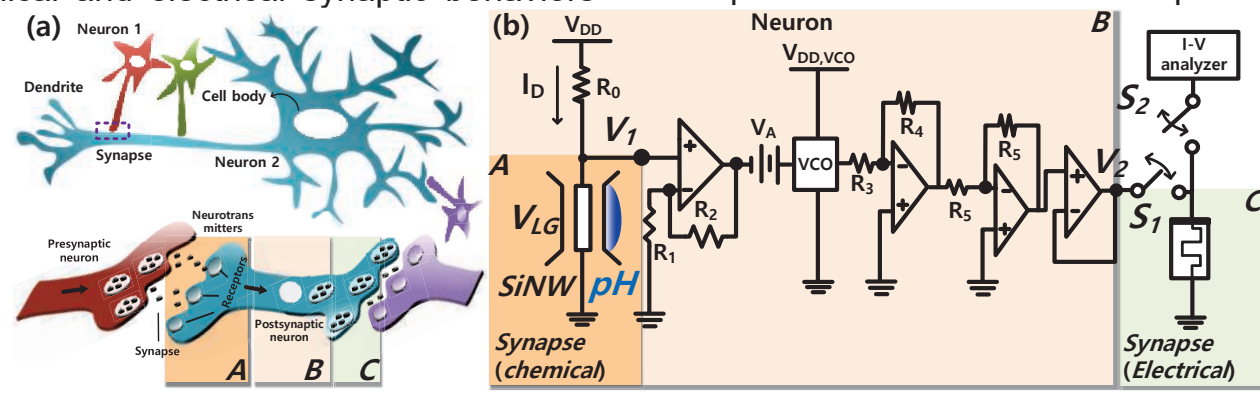

Fig.1. (a) Biological synapse and neuron. (b) Artificial synapse and neuron. 


\section{Result and Discussion}

The SiNW ISFET, IGZO memristor, and neuron circuit were characterized using an I-V analyzer (Keithley-4200). Used voltages were $V_{D D}=2 \mathrm{~V}$, $\mathrm{V}_{G S}=1.4 \mathrm{~V}, \mathrm{~V}_{\mathrm{DD}, \mathrm{VCO}}=12 \mathrm{~V}$, and $\mathrm{V}_{\mathrm{A}}=10 \mathrm{~V}$. Resistors of $R_{0}, R_{1}, R_{2}, R_{3}, R_{4}$, and $R_{5}$ having the values $500 \mathrm{k} \Omega, 2 \mathrm{k} \Omega, 5 \mathrm{k} \Omega, 12 \mathrm{k} \Omega, 5 \mathrm{k} \Omega, 12$ $k \Omega$ were used. The $V_{1}$ is determined with varying the $\mathrm{pH}$-dependent ISFET current ID as:

$\mathrm{V}_{1}=\mathrm{V}_{\mathrm{DD}}-\mathrm{I}_{\mathrm{D}} \times \mathrm{R}_{0}$.

On the other hand, the current of IGZO memristor (Imem) shows the LTP and STD operation depending on the frequency/interval of programming pulse (Fig. 2).

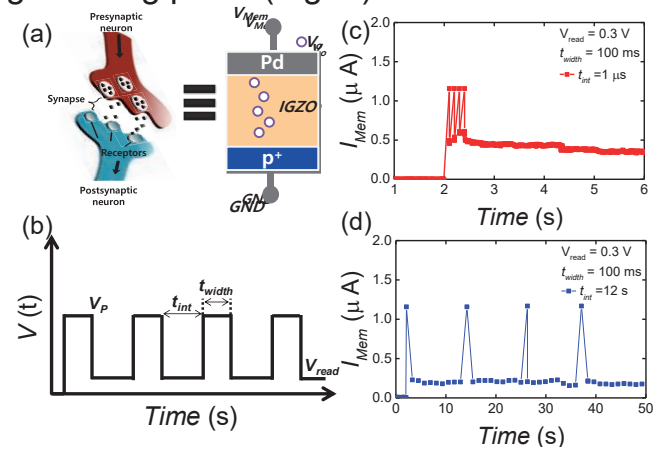

Fig. 2. (a) The electrical synapse based on IGZO memristor. (b) Pulse condition applied to memristor and the measured (c) LTP and (d) STD characteristics.

Measured $V_{1}$ is modulated with the variation of $\mathrm{pH}$ value (Fig. 3), which mimics the chemical synaptic behavior because this result suggests that the ISFET can transform the concentration of neurotransmitter agents into the input voltage $V_{1}$ of neuron circuit with the functionalization of their specific-binding receptors.
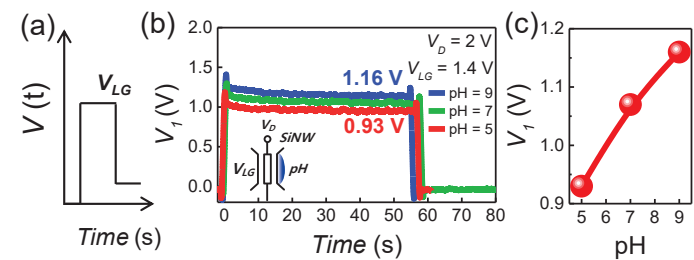

Fig. 3. (a) Time-varying condition of liquid gate voltage $V_{L G}$. (b) Measured time-varying $V_{1}$ (inset: the chemical synapse based on SiNW ISFET) and (c) the $\mathrm{pH}$-dependent $\mathrm{V}_{1}$.

The output voltage of VCO, i.e. $V_{2}$, successfully converts the concentration of hydrogen ions to the consecutive pulse trail with the $\mathrm{pH}$ dependent frequency (Fig. 4), which emulates the trail of output spikes out of post-synaptic neuron, i.e. the group of dendrite-cell body-axon, including the function of integration \& fire.

Finally, the post-synapse current (IPSC) is written to the IGZO memristor when the $S_{1}$ is closed and the $S_{2}$ is open and it is read when the $S_{2}$ is closed and the $S_{1}$ is open. The level of read IPSC is programmed depending on the $\mathrm{pH}$ value (Fig.
5), which duplicates the electrical synaptic operation, i.e. LTP and STD.
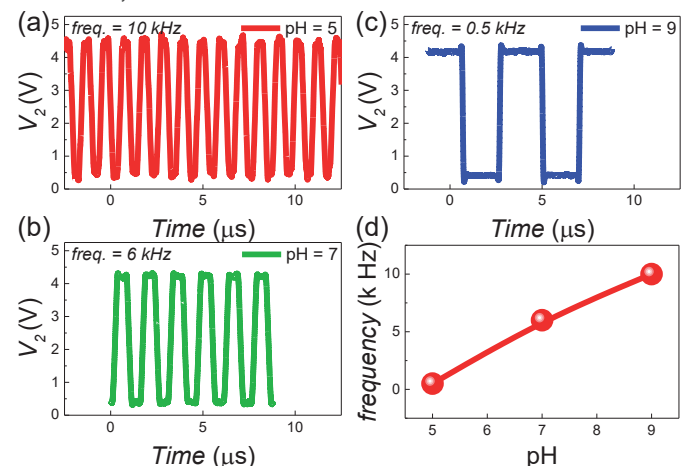

Fig. 4. Measured $\mathrm{V}_{2}$ when (a) $p H=5$, (b) $p H=7$, and (c) $\mathrm{pH}=9$. (d) The $\mathrm{pH}$-dependent frequency of $\mathrm{V}_{2}$.

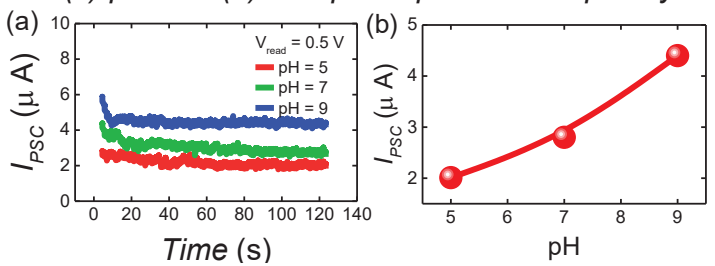

Fig. 5. (a) Measured IPSC when $V_{\text {read }}=0.5 \mathrm{~V}$. (b) The $\mathrm{pH}$-dependent IPSC.

\section{Conclusion}

Chemical and electrical operations of synapses were implemented by combining the $\mathrm{pH}$ sensing of ISFET and the LTP of IGZO memristor, respectively. The concentration of ions was successfully transformed via a simple neuron circuit block, into the modulation of the postsynapse current of memristor. Although they were not integrated, they did not directly detect neurotransmitters, nor did they use sophisticated neuron circuits, but the proposed artificial synapse and neuron can simulate the chemical and electrical behaviors of synapse at the same time. Therefore, our results are potentially useful for emulating the brain-inspired computing along with taking into accounts both chemical and electrical operation of synapse.

\section{Acknowledgment}

This work was supported by the National Research Foundation of Korea (NRF) grant funded by the Korea government (Ministry of Education, Science and Technology, MEST) (Nos. 2016R1A5A1012966, 2016R1A6A3A01006588, and 2016M3A7B4909668).

\section{References}

[1] T. Ohno, T. Hasegawa, T. Kazuya, J. K. Gimzewksi, M. Aono, Short-term plasticity and longterm potentiation mimicked in single inorganic synapses, Nat. Mater. 10, 591-595 (2011); doi: 10.1038/nmat3054.

[2] S. Thanapitak, C. Toumazou, A Bionics Chemical Synapse, IEEE Trans. on Bio. Circ. and Syst., 7, 296 - 306(2013); doi : 10.1109/TBCAS.2012.2202494

[3] J. Lee, J. Jang, B. Choi, J. Yoon, J.-Y. Kim, Y.-K. Choi, D. M. Kim, D. H. Kim, S.-J. Choi, A Highly Responsive Silicon Nanowire/Amplifier MOSFET Hybrid Biosensor, Scientific Rep. 5 (2015) doi: 10.1038/srep12286 